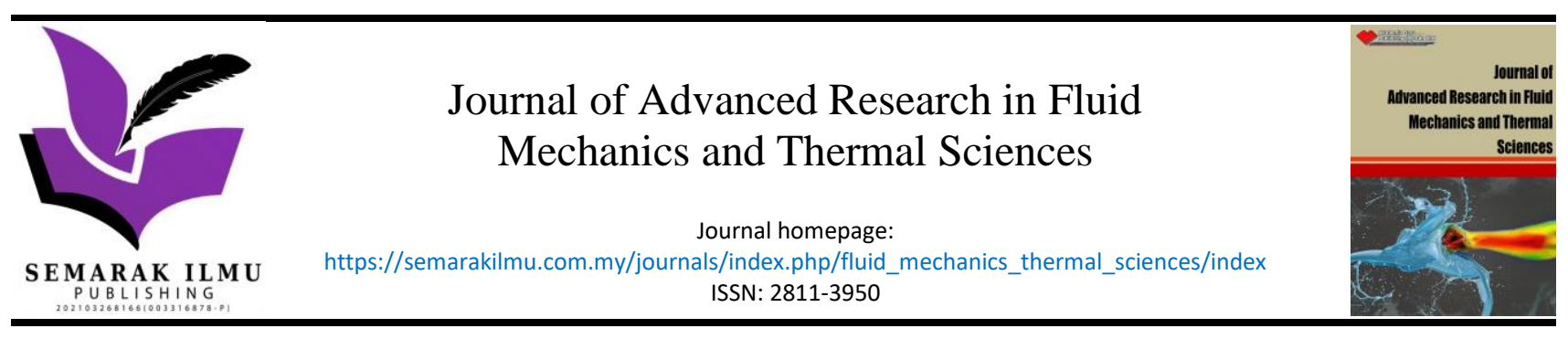

\title{
A Study of MHD Free Convection Flow Past an Infinite Inclined Plate
}

\author{
Husna Izzati Osman ${ }^{1}$, Nur Fatihah Mod Omar ${ }^{1}$, Dumitru Vieru², Zulkhibri Ismail ${ }^{1, *}$ \\ 1 Centre for Mathematical Sciences, College of Computing and Applied Sciences, Universiti Malaysia Pahang, Lebuhraya Tun Razak, 26300 \\ Gambang, Pahang, Malaysia \\ 2 Department of Theoritical Mechanics (MT), Georgia Asachi Technical University of lasi TU lasi, Romania
}

\section{ARTICLE INFO ABSTRACT}

\section{Article history:}

Received 5 August 2021

Received in revised form 14 December 2021

Accepted 21 December 2021

Available online 29 January 2022

\section{Keywords:}

Radiation; magnetohydrodynamics;

accelerated plate; Casson fluid

\begin{abstract}
In the present article, we have studied the magnetohydrodynamic (MHD) free convection flow past an infinite inclined plate. In order to explore the effects of velocity, temperature and concentration, Laplace transform technique has been used in this study. This method is adapted to accomplish the analytical solution of governing equation. The impact of various embedded parameter on concentration, velocity and temperature such as chemical reaction, magnetic parameter, radiation and inclination angle has been discussed graphically with numerical results.
\end{abstract}

\section{Introduction}

Magnetohydrodynamics (MHD) free convection flows have important applications in the field of planetary magneto-sphere, aeronautics and chemical engineering. Hannes Alfven was the first person who initiated the field of MHD and he successfully received the Nobel Prize in Physics in 1970. Therefore, many researchers inspired to discover the problems of MHD free convection flows with heat and mass transfer through various domains with the different boundary conditions. Basically, MHD is the study of the magnetic properties and behaviour of electrically conducting fluids that involved the magnetofluids such as plasmas, liquid, metal, salt, water and electrolytes. Alim et al., [1] interested to study about the effect of MHD natural convection flow along a vertical flat plate with Joule heating and heat conduction. This work transform the governing boundary layer equations into non-dimensional form and resulting nonlinear system of partial different equations are then solved numerically by using the implicit finite difference method with Keller box scheme. The couple effect of natural convection and conduction required the heat flux and temperature be continous at the interface. They finally drawn the conclusion from the present investigation. Both of the velocity distribution and the temperature distribution are increased because the Joule heating parameter increased while velocity decreases and temperature increased and it is affected to skin friction.

\footnotetext{
* Corresponding author.

E-mail address: zulkhibri@ump.edu.my
}

https://doi.org/10.37934/arfmts.92.1.1827 
Bulinda et al., [2] take an opportunity to study MHD free convection of incompressible fluids over corrugated vibrating bottom surface with Hall currents and heat mass transfer considering heat flux. Corrugated structures are well known for heat transfer enhancement applied in many engineering problems such as heat exchangers, air-conditioning devices and refrigeration. They conclude that the velocity profiles decreases with an increase of Prandtl number, Schmidt number and magnetic parameter. Meanwhile, Sharma et al., [3] has research about the unsteady free convection flow with heat and mass transfer of an electrically conducting viscoelastic fluid, through a porous medium of variable permeability. This research is different with Bulinda et al., [2] where they focused on the half space of fluid domain, bounded by the vertical porous plate with the constant heat flux, constant concentration and a rectilinear translation in its plane with constant velocity. They observed that the permeability with respect to time decreases, lead to the slower fluid movement and the fluid movement is faster due to the delta parameter increase.

A very little attention from the researchers to the problem of natural convection over an inclined plate makes Raju et al., [4] studied about the unsteady free convection boundary layer flow past a peridiocally accelerated vertical plate with Newtonian heating. It is observed that the temperature increase as time increase and the become decreases in the presence of Prandtl number. The boundary layer become thicker as thermal conductivity increase. Ismail et al., [5] are attracted to study the combined effects of thermal radiation and mass transfer on unsteady MHD free convection flow in a porous medium past an infinite inclined plate with constant temperature. The governing partial differential equations have been solved analytically by using laplace transform technique. They observed that the fluid velocity increase as the radiation parameter depleted. This is explained by te fact that the rate of transportation energy to the fluid increases when the radiation parameter decreases.

Khan et al., [6] were investigated the effects of an arbitrary wall shear stress on unsteady MHD flow of a Newtonian fluid in a porous medium over an inclined plate with ramped temperature with conjugate effects of heat and mass transfer. By taking velocity as an element to be discussed, they have found the velocity increases with the increment of Grashof number due to thermal bouyancy force tends to accelerate velocity for both ramped temperature and isothermal temperature. Different with Khan et al., [6] the influence of the inclination of the plate on the mixed convection heat transfer of a Casson rheological fluid past an inlcined plate using boundary theory has been investigated by Rao et al., [7]. From this research, they have found that, the temperature profiles consistenly decay from a maximum at the inclined plate surface to the free stream. Temperature also rise with the decreasing of shear stress and this situation allows more effective of transfer heat from the wall to the fluid regime. Palani et al., [8] are interested to analysis about the effects of MHD on two -dimenisonal free-convective flow of a viscous incompressible fluid past a semi-infinite isothermal inclined plate. Based on their result, they stated that when inclination angle increase, the normal component of the buoyancy force decreases near the leading edge which lead an implusive driving force for fluid motion along the plate.

Influence of chemical reaction with heat source under the study of convective flow with heat and mass transfer plays an important role in many areas in science and engineering field. Rout et al., [9] decided to research about the influence of chemical reaction and the combined effects of internal heat generation and a convective boundary condition on the laminar boundary layer MHD heat and mass transfer flow over a moving vertical flat plate. Hari et al., [10] has analysed the effects of chemical reaction on MHD mixed convection with the stagnation pount flow towards a vertical plate embedded in a porous medium with radiation and internal heat generation. The meaning of stagnation point in the present work is a point in a flow field where the local velocity of fluid is zero. 
Meanwhile, Osman et al., [11] interested to study about the chemical reaction and radiation effects on unsetady MHD free convection flow in a porous medium past an infinite inclined isothermal plate.

This research is focused into natural convection within Newtonian fluid such as water, oil gasoline and alcohol. The problem is limited to heat and mass transfer through inifinite inclined isothermal plate because from previous study, no one yet has started to research about MHD free convection flow past an infinite incline plate.

\section{Mathematical Formulation and Solution}

Consider the unsteady MHD free convection flow of an incompressible viscous, electrically conducting heat fluid near an infinite incliuned plate. With the reference to the coordinate system plate ${ }_{x}{ }^{*}$-axis along to the plate with an inclination angle $\phi$ to the vertical plate, the $y *$-plane. The motion of the fluid is governed by the following constitutive equation

$$
\begin{aligned}
& \frac{\partial u^{*}}{\partial t^{*}}=v \frac{\partial^{2} u^{*}}{\partial y^{* 2}}-\frac{\sigma B_{0}^{2}}{\rho} u^{*}-\frac{v}{K^{*}} u^{*}+g \beta_{T} \cos \phi\left(T^{*}-T_{\infty}^{*}\right)+g \beta_{C} \cos \phi\left(C^{*}-C_{\infty}^{*}\right) \\
& \frac{\partial T^{*}}{\partial t^{*}}=\frac{k}{\rho c_{p}} \frac{\partial^{2} T^{*}}{\partial y^{* 2}}-\frac{1}{\rho c_{p}} \frac{\partial q_{r}}{\partial y} \\
& \frac{\partial C^{*}}{\partial t^{*}}=D \frac{\partial^{2} C^{*}}{\partial y^{* 2}}-K_{r}\left(C^{*}-C_{\infty}^{*}\right)
\end{aligned}
$$

where $u^{*}, T^{*}, C^{*}$ represent velocity, temperature and concentration respectively, $v$ is kinematic viscosity, $g$ is acceleration due to gravity, $\sigma$ is the electrical conductivity $\rho$ is the fluid density, $K^{*}>0$ is the permeability of the porous medium, $\beta_{T}$ and $\beta_{C}$ are the thermal expansion and concentration expansion, $k$ is the thermal conductivity, $c_{p}$ is the specific heat, $q_{r}$ is the radiative heat flux, $D$ is the mass diffusion and $K_{r}$ is the chemical reaction parameter.

Initially, at time $t^{*} \leq 0$, the plate and the fluid are at rest with the same temperature $T_{\infty}^{*}$ and concentration $C_{\infty}^{*}$. At time $t^{*}>t_{0}$, both plate of temperature and concentration is raised to constant temperature $T_{w}^{*}$ and constant concentration $C_{w}^{*}$ while when $t^{*}>0$, both plate of temperature and concentration are approaching to zero. The following condition explain the appropriate boundary equations:

$$
\begin{aligned}
& u^{*}=0, T^{*}=T_{\infty}^{*}, C^{*}=C_{\infty}^{*} \text { for } y^{*} \geq 0 \text { and } t^{*} \leq 0 \\
& u^{*}=0, T^{*}=T_{w}^{*}, C^{*}=C_{w}^{*} \text { for } y^{*}=0 \text { and } t^{*}>t_{0} \\
& u^{*} \rightarrow 0, T^{*} \rightarrow 0, C^{*} \rightarrow 0 \text { for } y^{*} \rightarrow \infty \text { and } t^{*}>0
\end{aligned}
$$

According to the governing equations, temperature of the plates, $T_{\infty}^{*}$ and $T_{w}^{*}$, is assumed to produced radiative heat flux term and simplified by using Rosseland approximation (Mohan et al., [12] ) is given by 
$q_{r}=-\frac{4 \sigma^{*}}{3 k^{*}} \frac{\partial T^{* 4}}{\partial y^{*}}$

where $\sigma^{*}$ is Stefan-Boltzmann constant and $k^{*}$ is the mean absorption coefficient. We assume that the temperature differences within the flow are sufficiently small such that $T^{* 4}$ may be expressed as a linear function of the temperature. Using Taylor series by expanding $T^{* 4}$ about $T_{\infty}^{*}$ and neglecting higher-order terms, thus

$$
T^{* 4} \cong 4 T_{\infty}^{3 *} T-3 T_{\infty}^{4}
$$

Rosseland approximation become,

$$
q_{r}=-\frac{16 \sigma^{*} T_{\infty}^{3^{*}}}{3 K^{*}} \frac{\partial^{2} T^{* 4}}{\partial y^{* 2}}
$$

The dimensionless variable are introduce as follows:

$$
y=\frac{y^{*}}{L}, \quad t=\frac{t^{*}(v g)^{1 / 3}}{L}, u=\frac{u^{*}}{(v g)^{1 / 3}}, \quad T=\frac{T^{*}-T_{\infty}^{*}}{T_{w}^{*}-T_{\infty}^{*}}, \quad C=\frac{C^{*}-C_{\infty}^{*}}{C_{w}^{*}-C_{\infty}^{*}}
$$

Based on the Eq. (1), Eq. (2) and Eq. (3), the governing equations of momentum, energy and concentration are reduced by using Eq. (8) into following non-dimensional forms:

$$
\begin{aligned}
& \frac{\partial u}{\partial t}=\frac{\partial^{2} u}{\partial y^{2}}-M u-\frac{u}{K}+G r T \cos \phi+G c C \cos \phi \\
& \frac{\partial T}{\partial t}=\frac{(1+R)}{P e} \frac{\partial^{2} T}{\partial y^{2}} \\
& \frac{\partial C}{\partial t}=\frac{1}{P e_{c}} \frac{\partial^{2} C}{\partial y^{2}}-K r C
\end{aligned}
$$

where

$$
\begin{aligned}
& M=\frac{\sigma B_{0}^{2} L^{2}}{\rho v}, K=\frac{K^{*}}{L^{2}}, G r=\frac{g^{1 / 3} \beta_{T}\left(T_{w}^{*}-T_{\infty}^{*}\right) L}{v^{2 / 3}}, G c=\frac{g^{1 / 3} \beta_{C}\left(C_{w}^{*}-C_{\infty}^{*}\right) L}{v^{2 / 3}}, R=\frac{16 \alpha \sigma^{*} T_{\infty}^{*} L^{2}}{k}, \\
& P e=\operatorname{RePr}, P e_{c}=\operatorname{Re} S c
\end{aligned}
$$

where, $M$ is magnetic parameter known as Hartmann number, $K$ is the porosity parameter, $G r$ is the thermal Grasof number, $G c$ is the mass Grasof number, $R$ is radiation parameter $P e$ known as Peclet's number of mass transfer, $P e_{c}$ is known as Peclet's number of concentration, Re is Reynold number, Pr is Prandtl number and $S c$ is Schmidt number. The characteristic length can be defined as: 


$$
L=\frac{v^{2 / 3}}{g^{1 / 3}}
$$

The dimensionless initial and boundary conditions become:

$$
\begin{aligned}
& u=0, T=0, C=0 \text { for } y \geq 0 \text { and } t \leq 0 \\
& u=0, T=1, C=1 \text { for } y=0 \text { and } t \geq 0 \\
& u \rightarrow 0, T \rightarrow 0, C \rightarrow 0 \text { for } y \rightarrow \infty \text { and } t>0
\end{aligned}
$$

\section{Solution of the Problem}

Laplace transform technique has been applied in order to solve the equations. Hence, the temperature variable $T(y, t)$ and concentration variable $C(y, t)$ can be solve where the solution $u(y, t)$ can be achieved. The energy Eq. (2) and concentration Eq. (3) is uncoupled from the momentum Eq. (1). Eq. (9), Eq. (10) and Eq. (11) are solved by using laplace transform with respect to $t$ with the present of Eq. (13) and solving the result from different equations, we achieved:

Energy Equation:

$$
\bar{T}=\frac{1}{S} e^{-y \sqrt{\frac{s\left(P e_{m}\right)}{1+R}}}
$$

Concentration Equation:

$$
\bar{C}=\frac{1}{S} e^{-y \sqrt{P e_{c}(K r+s)}}
$$

Momentum Equation:

$$
\begin{aligned}
& \bar{u}=a_{1}\left[\frac{1}{a_{2}\left(s-a_{2}\right)}-\frac{1}{a_{2} s}\right] e^{-y \sqrt{\frac{s(\mathrm{RePr})}{(1+R)}}}+a_{3}\left[\frac{1}{a_{4}\left(s-a_{4}\right)}-\frac{1}{a_{4} s}\right] e^{-y \sqrt{(K r+s) \mathrm{Re} S c}} \\
& a_{1}\left[\frac{1}{a_{2}\left(s-a_{2}\right)}-\frac{1}{a_{2} s}\right] e^{-y \sqrt{\lambda+s}}-a_{3}\left[\frac{1}{a_{4}\left(s-a_{4}\right)}-\frac{1}{a_{4} s}\right] e^{-y \sqrt{\lambda+s}}
\end{aligned}
$$

where

$$
a_{1}=\frac{-G r \cos \phi}{\left(\frac{P e}{1+R}-1\right)}, a_{2}=\frac{\lambda(1+R)}{P e-1}, a_{3}=\frac{-G c \cos \phi}{(1-\operatorname{Re} S c)}
$$

Therefore, the exact solution for the temperature, concentration and velocity are obtained from Eq. (15), Eq. (16), Eq. (17) by using inverse Laplace transform. These solutions are: 


$$
\begin{aligned}
& T=\operatorname{erfc}\left(\frac{y}{2} \sqrt{\frac{\theta}{t}}\right) \\
& C=\frac{1}{2}\left[e^{y \sqrt{\operatorname{Re} S c K r}} \operatorname{erfc}\left(\frac{y}{2} \sqrt{\frac{\operatorname{Re} S c}{t}}\right)+\sqrt{K r t}+e^{-y \sqrt{\operatorname{Re} S c K r}} \operatorname{erfc}\left(\frac{y}{2} \sqrt{\frac{\operatorname{Re} S c}{t}-\sqrt{K r t}}\right)\right] \\
& u_{1}(y, t)=\frac{a_{1} e^{a_{2} t}}{2 a_{2}}\left[e^{y \sqrt{\theta a_{2}}} \operatorname{erfc}\left(\frac{y}{2} \sqrt{\frac{\theta}{t}}+\sqrt{a_{2} t}\right)+e^{-y \sqrt{\theta a_{2}}} \operatorname{erfc}\left(\frac{y}{2} \sqrt{\frac{\theta}{t}}-\sqrt{a_{2} t}\right)\right] \\
& u_{2}(y, t)=\frac{a_{1}}{a_{2}} \operatorname{erfc}\left(\frac{y}{2} \sqrt{\frac{\operatorname{Pr}}{t}}\right) \\
& u_{3}(y, t)=\frac{a_{3} e^{a_{4} t}}{2 a_{4}}\left[e^{y \sqrt{P e_{m}\left(K r+a_{4}\right)}} \operatorname{erfc}\left(\frac{y}{2} \sqrt{\frac{P e_{m}}{t}}+\sqrt{\left(K r+a_{4}\right) t}\right)+e^{-y \sqrt{P e_{m}\left(K r+a_{4}\right)}} \operatorname{erfc}\left(\frac{y}{2} \sqrt{\frac{P e_{m}}{t}}-\sqrt{\left(K r+a_{4}\right) t}\right)\right] \\
& u_{4}(y, t)=\frac{a_{3}}{2 a_{4}}\left[e^{y \sqrt{P e_{m} K r}} \operatorname{erfc}\left(\frac{y}{2} \sqrt{\frac{P e_{m}}{t}}+\sqrt{K r t}\right)+e^{-y \sqrt{P e_{m} K r}} \operatorname{erfc}\left(\frac{y}{2} \sqrt{\frac{P e_{m}}{t}}-\sqrt{K r t}\right)\right] \\
& u_{5}(y, t)=\frac{a_{1} e^{a_{2} t}}{2 a_{2}}\left[e^{y \sqrt{\left.\lambda+a_{4}\right)}} \operatorname{erfc}\left(\frac{y}{2 \sqrt{t}}+\sqrt{\left(\lambda+a_{2}\right) t}\right)+e^{-y \sqrt{\lambda+a_{2}}} \operatorname{erfc}\left(-\frac{y}{2 \sqrt{t}}-\sqrt{\left(\lambda+a_{2}\right) t}\right)\right] \\
& u_{6}(y, t)=\frac{a_{1}}{2 a_{2}}\left[e^{y \sqrt{\lambda)}} \operatorname{erfc}\left(\frac{y}{2 \sqrt{t}}+\sqrt{\lambda t}\right)+e^{-y \sqrt{\lambda}} \operatorname{erfc}\left(\frac{y}{2 \sqrt{t}}-\sqrt{\lambda t}\right)\right] \\
& u_{7}(y, t)=\frac{a_{3} e^{a_{4} t}}{2 a_{4}}\left[e^{y \sqrt{\left.\lambda+a_{4}\right)}} \operatorname{erfc}\left(\frac{y}{2 \sqrt{t}}+\sqrt{\left(\lambda+a_{4}\right) t}\right)+e^{-y \sqrt{\left.\lambda+a_{4}\right)}} \operatorname{erfc}\left(-\frac{y}{2 \sqrt{t}}-\sqrt{\left(\lambda+a_{4}\right) t}\right)\right] \\
& u_{8}(y, t)=\frac{a_{3}}{2 a_{4}}\left[e^{y \sqrt{\lambda}} \operatorname{erfc}\left(\frac{y}{2 \sqrt{t}}+\sqrt{\lambda t}\right)+e^{-y \sqrt{\lambda}} \operatorname{erfc}\left(\frac{y}{2 \sqrt{t}}-\sqrt{\lambda t}\right)\right]
\end{aligned}
$$

$\operatorname{erfc}(x)$ being the complimentary error function defined by

$$
\operatorname{erfc}(x)=1-\operatorname{erf}(x), \operatorname{erf}(x) \frac{2}{\sqrt{n}} \int_{0}^{x} e^{-\eta^{2}} d \eta
$$

\section{Graphical Result and Discussion}

In this section, the results discussed in the previous section are presented through graph. The effect of various physical parameter such as magnetic field, inclination plate, radiation and chemical reaction on the convection flow, the graph of velocity and temperature are obtained. All the discussion about the graph has been made. 
The velocity profile of magnetic field $M$ parameter is shown in Figure 1. From the graph,we can observe that the velocity decreasing when the values of magnetic field increased. This is happened due to the application of the tranverse magnetic field that give a resistive type of force called Lorentz force. The velocity is reduced because of the Lorentz force which it tends to resist the fluid flow.

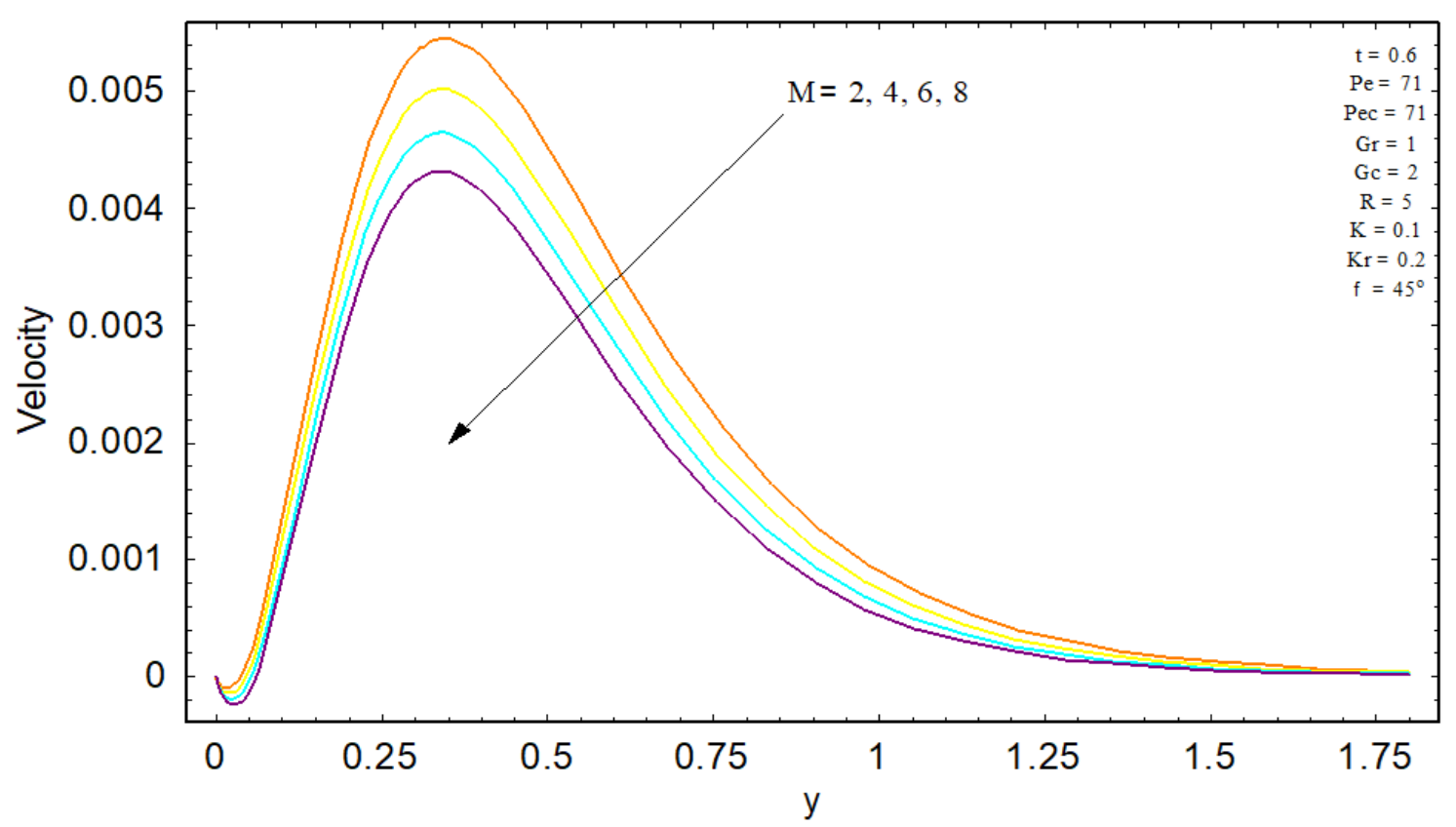

Fig. 1. The influence of magnetic field on the velocity profiles

Figure 2 shows the velocity profiles at various value of inclination angle $\phi$. It shows that the increasing of inclination angle accelerates the fluid motion along the plate. This may be explained that as the plate is inclined from the vertical, the bouyancy force is affected. This is due to the thermal and mass diffusion reduced as $\cos \phi$ decreased.

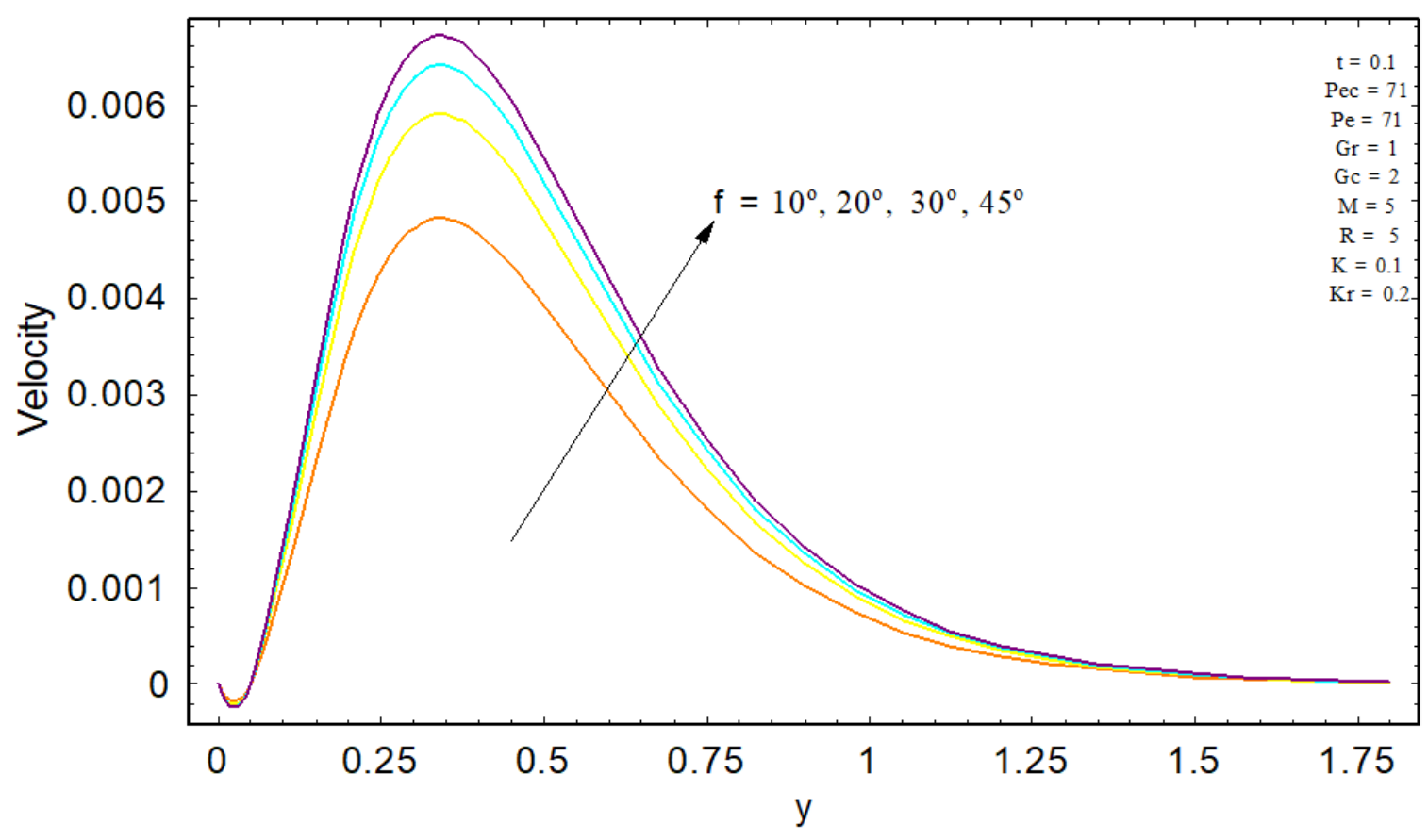

Fig. 2. The influence of inclination angle on the velocity profiles 
Figure 3 depicts the influence of temperature profiles at various value of radiation parameter. The value of time $t$ of the temperature profile is set to be 0.6. From this figure, we observed that the temperature of the fluid increases as the radiation parameter increases. Existence of radiation are affected the thermal boundary layer to become thicker since the radiation provides an additional means to diffuse energy.

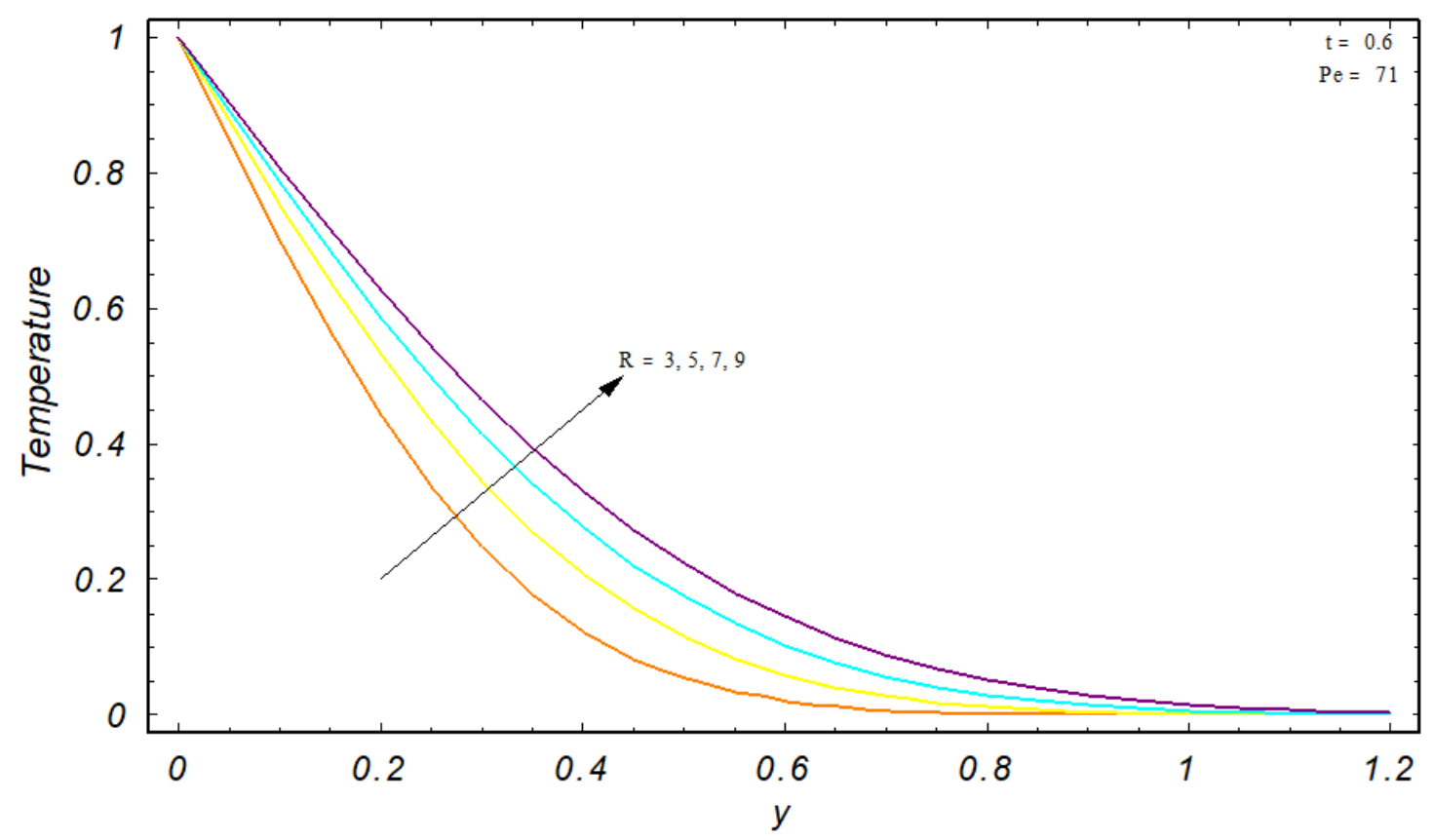

Fig. 3. The influence of radiation on the velocity profiles

The temperature profiles at various chemical reaction parameter $K r$ is presented in Figure 4 . As we can see from the graph, the increasing value of $K r$ has affecting the concentration to become decreased.

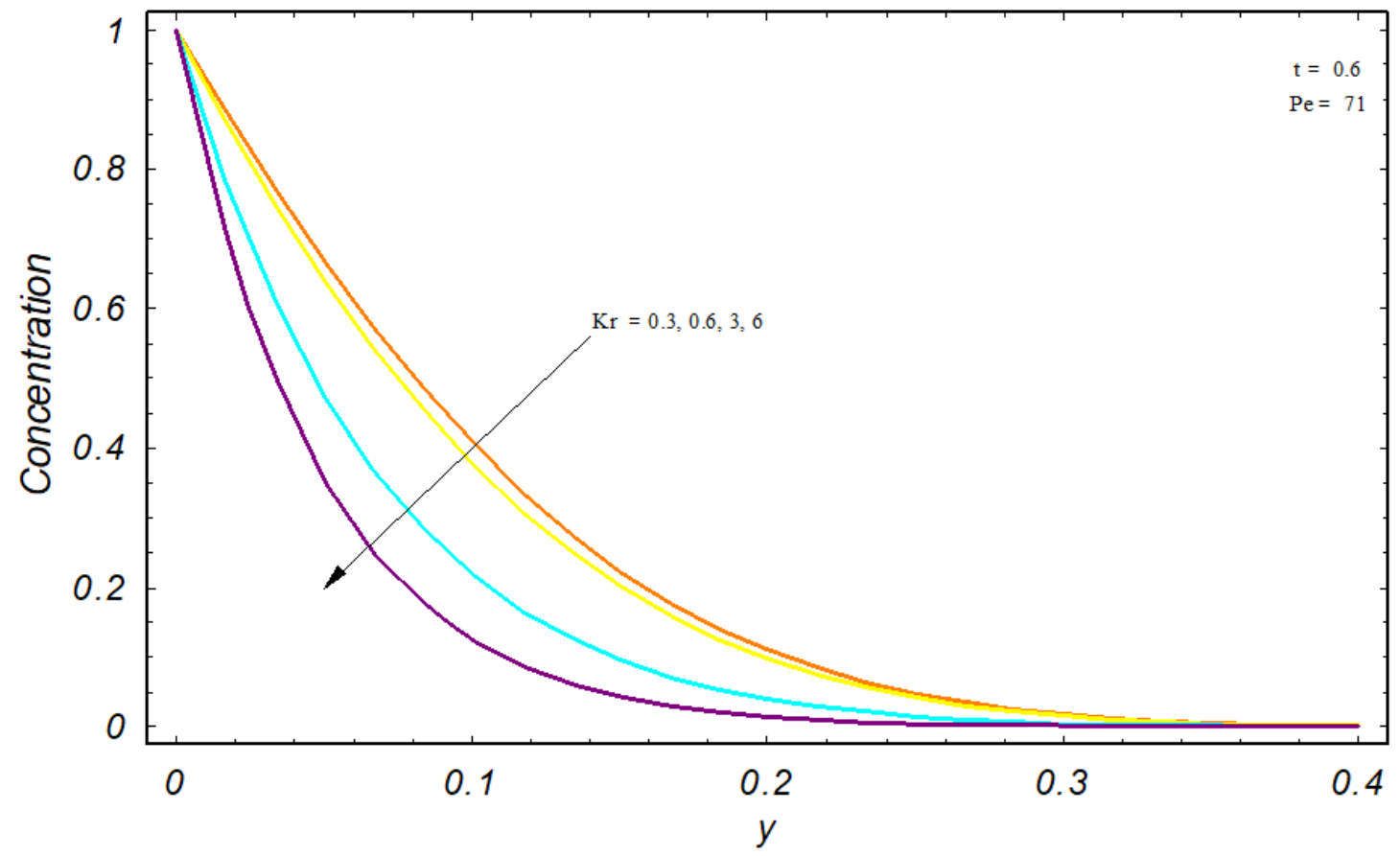

Fig. 4. The influence of chemical reaction on the concentration profiles 


\section{Conclusions}

This research was conducted to solve the problem of magnetohydrodynamic free convection flow past an inclined plate including magnteic parameter, inclination angle, radiation and chemical reaction. Laplace transform technique has been using throughout the research to solve the governing equation. The result of various parameter embedded on the velocity, temperature and concentration are shown in the graph whereas skin-friction is shown in table. The conclusion of the result from graph has been made where the velocity is reduced when the values of magnetic parameter had an increment. Resistive type of force named Lorentz force is produced due to the application of tranverse magentic filed in this result.

Besides, it is observe that the increment value of inclination angle accelerates the fluid motion along the plate. Bouyancy force effected when the plate is inclined from the vertical. The trend distribution of temperature at various value of radiation shows the temperature increase with the increasing of radiation parameter since radiation provide additional means to diffuse energy and it is affected the thermal boundary layer become thicker. Based on the concentration profile of chemical recation parameter, the result shows the concentration id reduced when the $K r$ value increased.

\section{Acknowledgement}

The authors would like to acknowledge UMP's Centre for Research in Advanced Fluid \& Processes (RDU190376) and UMP Internal Postgraduate Research Grant (PGRS210329) for this research.

\section{References}

[1] Alim, M. A., Md M. Alam, and Abdullah Al-Mamun. "Joule heating effect on the coupling of conduction with magnetohydrodynamic free convection flow from a vertical flat plate." Nonlinear Analysis: Modelling and Control 12, no. 3 (2007): 307-316. https://doi.org/10.15388/NA.2007.12.3.14688

[2] Bulinda, Vincent M., Giterere P. Kang'ethe, and Phineas R. Kiogora. "Magnetohydrodynamics free convection flow of incompressible fluids over corrugated vibrating bottom surface with hall currents and heat and mass transfers." Journal of Applied Mathematics 2020 (2020). https://doi.org/10.1155/2020/2589760

[3] Sharma, P. R., Navin Kumar, and Pooja Sharma. "Influence of chemical reaction and radiation on unsteady MHD free convection flow and mass transfer through viscous incompressible fluid past a heated vertical plate immersed in porous medium in the presence of heat source." Appl. Math. Sciences 5, no. 46 (2011): 2249-2260.

[4] Raju, M. C., S. V. K. Varma, and A. J. Chamkha. "Unsteady free convection flow past a periodically accelerated vertical plate with Newtonian heating." International Journal of Numerical Methods for Heat \& Fluid Flow 26, no. 7 (2016): 2119-2138. https://doi.org/10.1108/HFF-05-2014-0123

[5] Ismail, Zulkhibri, Ilyas Khan, Nadirah Mohd Nasir, Rahimah Jusoh, Mohd Zuki Salleh, and Sharidan Shafie. "Rotation effects on coupled heat and mass transfer by unsteady MHD free convection flow in a porous medium past an infinite inclined plate." In AIP Conference Proceedings, vol. 1605, no. 1, pp. 410-415. American Institute of Physics, 2014. https://doi.org/10.1063/1.4887624

[6] Khan, Arshad, Ilyas Khan, Farhad Ali, and Sharidan Shafie. "Effects of wall shear stress on MHD conjugate flow over an inclined plate in a porous medium with ramped wall temperature." Mathematical Problems in Engineering 2014 (2014). https://doi.org/10.1155/2014/861708

[7] Rao, A. Subba, V. Ramachandra Prasad, N. Nagendra, N. Bhaskar Reddy, and O. Anwar Beg. "Non-Similar Computational Solution for Boundary Layer Flows of Non-Newtonian Fluid from an Inclined Plate with Thermal Slip." Journal of Applied Fluid Mechanics 9, no. $2 \quad$ (2016): $795-807$. https://doi.org/10.18869/acadpub.jafm.68.225.24664

[8] Palani, G., and Kwang-Yong Kim. "The effects of MHD on free-convection flow past a semi-infinite isothermal inclined plate." Journal of Engineering Physics and Thermophysics 81, no. $4 \quad$ (2008): $724-731$. https://doi.org/10.1007/s10891-008-0087-3

[9] Rout, B. R., S. K. Parida, and S. Panda. "MHD heat and mass transfer of chemical reaction fluid flow over a moving vertical plate in presence of heat source with convective surface boundary condition." International Journal of Chemical Engineering 2013 (2013). https://doi.org/10.1155/2013/296834 
[10] Hari, Niranjan, S. Sivasankaran, M. Bhuvaneswari, and Zailan Siri. "Effects of chemical reaction on MHD mixed convection stagnation point flow toward a vertical plate in a porous medium with radiation and heat generation." In Journal of Physics: Conference Series, vol. 662, no. 1, p. 012014. IOP Publishing, 2015. https://doi.org/10.1088/1742-6596/662/1/012014

[11] Osman, H. I., N. F. M. Omar, D. Vieru, and Z. Ismail. "Chemical reaction and radiation effects on unsteady MHD free convection flow in a porous medium past an infinite inclined isothermal plate." Data Analytics and Applied Mathematics (DAAM) 1, no. 1 (2020): 01-10. https://doi.org/10.15282/daam.v1i01.5082

[12] Mohan, S. Rama, Prof. G. Viswanatha Reddy and Subash Balakrishna. "An Unsteady MHD Free Convection Flow of Casson Fluid Past an Exponentially Accelerated Infinite Vertical Plate Through Porous Media in The Presence of Thermal Radiation, Chemical Reaction and Heat Source or Sink." (2018). https://doi.org/10.1166/asem.2019.2355

[13] Lavanya, Bommanna, and Madduleti Nagashashikala. "Effects of chemical reaction and heat generation on the unsteady free convection flow past an infinite vertical permeable moving plate with variable temperature." Journal of Advanced Research in Fluid Mechanics and Thermal Sciences 64, no. 2 (2019): 244-252.

[14] Qin, Y. Z., A. N. Darus, and NA Che Sidik. "Numerical Analysis on Natural Convection Heat Transfer of a Heat Sink with Cylindrical Pin Fin." Journal of Advanced Research in Fluid Mechanics and Thermal Sciences 2, no. 1 (2014): 1322.

[15] Saidin, Norshaza Atika, Mohd Ariff Admon, and Khairy Zaimi. "Unsteady Three-Dimensional Free Convection Flow Near the Stagnation Point Over a General Curved Isothermal Surface in a Nanofluid." CFD Letters 12, no. 6 (2020): 80-92. https://doi.org/10.37934/cfdl.12.6.8092

[16] Khan, Ansab Azam, Khairy Zaimi, Suliadi Firdaus Sufahani, and Mohammad Ferdows. "MHD Mixed Convection Flow and Heat Transfer of a Dual Stratified Micropolar Fluid Over a Vertical Stretching/Shrinking Sheet With Suction, Chemical Reaction and Heat Source." CFD Letters 12, no. $11 \quad$ (2020): 106-120. https://doi.org/10.37934/cfdl.12.11.106120

[17] Narahari, M. "An Exact Solution of Unsteady MHD Free Convection Flow of a Radiating Gas Past an Infinite Inclined Isothermal Plate." Applied Mechanics and Materials $110 \quad$ (2011): 38-49. https://doi.org/10.4028/www.scientific.net/AMM.110-116.2228

[18] Molla, Md Mamun, Suvash C. Saha, and Md Anwar Hossain. "Radiation effect on free convection laminar flow along a vertical flat plate with streamwise sinusoidal surface temperature." Mathematical and Computer Modelling 53, no. 5-6 (2011): 1310-1319. https://doi.org/10.1016/i.mcm.2010.12.017

[19] Pattnaik, Jyotsna Rani, Gouranga Charan Dash, and Suprava Singh. "Radiation and mass transfer effects on MHD flow through porous medium past an exponentially accelerated inclined plate with variable temperature." Ain Shams Engineering Journal 8, no. 1 (2017): 67-75. https://doi.org/10.1016/i.asej.2015.08.014

[20] Siddique, Imran, and Itrat Abbas Mirza. "Magneto-hydrodynamic free convection flows of a viscoelastic fluid in porous medium with variable permeability heat source and chemical reaction." Results in physics 7 (2017): 39283937. https://doi.org/10.1016/i.rinp.2017.09.054

[21] Ahmad, S., D. Vieru, I. Khan, and S. Shafie. "Unsteady magnetohydrodynamic free convection flow of a second grade fluid in a porous medium with ramped wall temperature." Plos one 9, no. 5 (2014): 1-9. https://doi.org/10.1371/journal.pone.0088766

[22] Balla, Chandra Shekar, and Kishan Naikoti. "Radiation effects on unsteady MHD convective heat and mass transfer past a vertical plate with chemical reaction and viscous dissipation." Alexandria Engineering Journal 54, no. 3 (2015): 661-671. https://doi.org/10.1016/j.aej.2015.04.013 